\title{
ESTRÉS OXIDATIVO EN LA RETINA DE LA RATA INDUCIDO POR LA ADMINISTRACIÓN CRÓNICA DE ETANOL: TRATAMIENTO CON EL ANTIOXIDANTE EBSELEN
}

\section{CHRONIC ETHANOL FEEDING INDUCES OXIDATIVE STRESS IN THE RAT RETINA: TREATMENT WITH THE ANTIOXIDANT EBSELEN}

\author{
JOHNSEN-SORIANO $\mathrm{S}^{1}$, GENOVÉS JM ${ }^{2}$, ROMERO B ${ }^{2}$, GARCÍA-DELPECH $\mathrm{S}^{2}$, MURIACH $\mathrm{M}^{3}$, \\ SANCHO-TELLO $\mathrm{M}^{2}$, BOSCH-MORELL $\mathrm{F}^{4}$, ROMERO FJ ${ }^{2}$
}

\section{RESUMEN}

Objetivo: Establecer la existencia de cambios bioquímicos y funcionales en la retina tras la administración crónica de etanol en ratas adultas, y estudiar la capacidad del antioxidante ebselen para corregir estos efectos.

Métodos: Se utilizaron ratas macho Sprague-Dawley, que fueron alimentadas con una dieta líquida con etanol, mientras el grupo control recibió una dieta isocalórica libre de etanol. Después de seis semanas, los ojos fueron extraídos y homogenizados sin cristalino, y se determinaron parámetros relevantes en la modulación del estrés oxidativo, tales como el contenido de glutation (GSH) y de malondialdehído (MDA) como antioxidante intracelular y producto de la peroxidación de lípidos, respectivamente. Además, se comprobó la funcionalidad de la retina mediante electrorretinograma (ERG).

\section{ABSTRACT}

Purpose: To assess the involvement of biochemical and functional changes to the retina after chronic ethanol intake in adult rats, and the capacity of the antioxidant ebselen to prevent these changes.

Methods: Male Sprague-Dawley rats were used in the study. They were fed an ethanol-containing liquid diet, whereas a control group was given an ethanol-free isocaloric diet. After six weeks of experiment, the eyes were extracted and homogenized without the lens, and markers of oxidative stress were assayed, i.e., glutathione (GSH) and malondialdehyde (MDA) as an intracellular antioxidant and a lipid peroxidation product, respectively. Moreover, retinal function was assessed by electroretinogram (ERG).

Results: The retinal MDA concentration was significantly increased in the ethanol-fed animals compared to controls, whereas the GSH content was

\footnotetext{
Recibido: 16/8/06. Aceptado: 17/9/07

1 Doctor en Biología.

2 Doctor en Medicina.

3 Doctor en Tecnología de los Alimentos.

4 Doctor en Farmacia.

Correspondencia:

F.J. Romero

Universidad CEU Cardenal Herrera

Avda. Seminari, s/n

46113 Moncada (Valencia)

España

E-mail: jromero@uch.ceu.es
}

Fundación Oftalmológica del Mediterráneo. Valencia. España.

Proyecto subvencionado por la Universidad Cardenal Herrera-CEU, la Dirección General de Drogodependencias de la Generalitat Valenciana y la FEPAD, Fundación para el Estudio, Prevención y Ayuda a la Drogadicción. 
Resultados: La concentración de MDA en la retina fue significativamente mayor en el grupo alimentado con etanol, mientras el contenido de GSH fue significativamente menor en este grupo, al compararlo con el grupo control. El etanol también indujo una disminución de la onda b del ERG. El tratamiento con ebselen fue capaz de corregir los valores de MDA, GSH y la amplitud de la onda b en el ERG hasta valores control.

Conclusión: Estos resultados indican que la ingesta crónica de etanol como único factor etiológico, altera el estado redox de la retina así como su función (ERG), descartando la influencia del estado nutricional. Aun así, son necesarios nuevos estudios para confirmar el mecanismo protector del ebselen en este modelo del alcoholismo crónico.

Palabras clave: Etanol, estrés oxidativo, glutation, peroxidación de lípidos, electrorretinograma, antioxidante, ebselen. significantly reduced in the ethanol-fed group compared to controls. Ethanol also induced a decrease in ERG b-wave amplitude. Ebselen treatment restored the MDA and GSH concentrations and ERG bwave amplitude to control values.

Conclusion: These results indicate that chronic alcohol consumption alone and without the influence of nutritional factors alters the retinal redox status as well as its function (ERG). Further studies are required to better understand the protective mechanism of ebselen in this experimental model of chronic alcoholism (Arch Soc Esp Oftalmol 2007; 82: 757-762).

Key words: Ethanol, oxidative stress, glutathione, lipid peroxidation, electroretinogram, antioxidant, ebselen.

\section{INTRODUCCIÓN}

El consumo crónico de etanol tiene una alta prevalencia en nuestra sociedad, pudiendo ejercer su acción tóxica por un efecto directo sobre la generación de radicales libres, o a través de sus metabolitos (1), fundamentalmente el acetaldehído (2). El estrés oxidativo se define como una situación de desequilibrio entre agentes oxidantes y antioxidantes en favor de los primeros (3). Se ha demostrado que la ingesta crónica de etanol conduce a un aumento de los productos de la peroxidación lipídica y a un descenso de factores antioxidantes (4) como el glutation (GSH) y sus enzimas relacionados (5). Los radicales libres son capaces de producir daños en distintos tejidos, y en la actualidad se investiga la posibilidad de utilizar antioxidantes como neuroprotectores en tejidos dañados por el etanol (6).

La retina es el tejido neurosensorial del ojo y sus membranas son extremadamente ricas en lípidos poliinsaturados. Esta característica la hace especialmente sensible a los radicales libres oxigenados y a la peroxidación lipídica (7). Varias enfermedades oculares se relacionan con el estrés oxidativo, tales como la retinopatía diabética y la uveítis $(8,9)$. Datos publicados previamente por nuestro laboratorio describen una disminución del contenido de GSH, así como una mayor concentración de malon- dialdehído (MDA) en el nervio óptico de ratas alcohólicas (10). Estos resultados junto a la importancia del estrés oxidativo en enfermedades retinianas y en el alcoholismo crónico, nos llevaron a investigar el papel de estos parámetros en la retina y ensayar el tratamiento con el antioxidante ebselen.

El ebselen es un compuesto seleno-orgánico biológicamente activo, con propiedades antiinflamatorias (11). Posee actividad similar a la glutation peroxidasa y se ha documentado su efecto como secuestrante de peroxinitritos (12). Actualmente se investiga el papel de este antioxidante en enfermedades como la retinosis pigmentaria, la retinopatía diabética y la uveítis.

\section{SUJETOS, MATERIAL Y MÉTODOS}

Para este estudio se utilizaron 24 ratas macho Sprague-Dawley (6 por grupo). La manipulación y cuidado de los animales se realizó siguiendo las normativas internacionales de la Comunidad Económica Europea (orden 86/609/CEE) y la de A.R.V.O. (Association for Research in Vision and Opthalmology).

Se dividieron las ratas, de 300-325 g, en 2 grupos de 12. Estos dos grupos se aparearon en función de su peso para el control de alimentación. El primer grupo (etanol) recibió como alimento la dieta Lie- 
ber-De Carli (13), una dieta utilizada habitualmente en modelos del alcoholismo crónico. El grupo etanol fue a su vez dividido en 2 grupos de 6 ratas, administrando ebselen a las ratas de uno de ellos junto con la dieta alcohólica $(0,1 \mathrm{mg} / \mathrm{ml}$ de dieta). Los valores de alcoholemia oscilaron entre 195-225 $\mathrm{mg} / \mathrm{dL}$ durante las últimas cinco semanas (análisis de sangre obtenida de la cola de animales seleccionados aleatoriamente durante todo este período, analizando dos animales por semana). El grupo control recibió una mezcla isocalórica de maltosa dextrinada sustituyendo al etanol, y fue dividido también en dos grupos de 6 animales, uno de los cuales recibió ebselen junto con la dieta.

Los animales recibieron la dieta durante 6 semanas y se mantuvieron, en jaulas individuales, en el estabulario del departamento de Fisiología, Farmacología y Toxicología de la Universidad Cardenal Herrera-CEU, en condiciones controladas de temperatura $\left(20^{\circ}\right)$ y humedad $(60 \%)$, bajo ciclos constantes de luz-oscuridad de 12 horas. Inmediatamente después del sacrificio de los animales, se enuclearon ambos ojos y se extrajo el cristalino. Las muestras se homogenizaron en tampón fosfato potásico 0,2 M, pH 7. Está demostrado que el contenido de antioxidantes en estas muestras corresponde a la retina en un $97 \%$ (14).

Para la cuantificación del MDA se determina el nivel del complejo formado entre el MDA con el ácido tiobarbitúrico, siguiendo una modificación del método de Richard (15) descrita por nuestro grupo (16), empleando un equipo de cromatografía líquida de alta resolución (HPLC, Waters). La concentración de GSH se determinó por una modificación del procedimiento descrito por Reed et al. (17), basado en la reacción del ácido iodoacético con los grupos tioles seguido de una derivatización cromófora de los grupos amino con el reactivo de Sanger (1-fluoro-2,4-dinitrobenceno) dando lugar a derivados que son rápidamente separados por HPLC, lo que permite la cuantificación de concentraciones nanomolares de GSH. El contenido de proteínas se determinó según el método propuesto por Lowry (18). El análisis estadístico se ha realizado según la prueba $\mathrm{t}$ de Student, considerándose significativa una $\mathrm{p}<0.05$. Los datos están expresados como media \pm desviación estándar (DE).

La medición del ERG se realizó a las ratas previamente anestesiadas con ketamina $(100 \mathrm{mg} / \mathrm{kg}$ peso) y azepromazina $(2,5 \mathrm{mg} / \mathrm{kg}$ peso $)$, y adaptadas a la oscuridad. Se administró un colirio anesté- sico y midriático. Se utilizó un electrodo de lazo de contacto conjuntival de oro, un electrodo de referencia en la nuca y un electrodo de masa en la cola de la rata. Los estímulos empleados son flashes con una duración máxima de 4 ms [media 2; rango 100; intensidad $1\left(0.06\right.$ x 22 lumen sec/ $\left.\left./ \mathrm{ft}^{2}\right)\right]$. Se coloca delante un flash blanco estándar con un filtro de 2,5 unidades logarítmicas de densidad óptica. La banda pasante del amplificador y preamplificador se establece en 3-50 Hz. Los registros se recogen en un equipo informático MacLab (Castle Hill, Australia). Se midió la amplitud de las ondas a y b del ERG.

\section{RESULTADOS}

El volumen medio de dieta ingerido por los diferentes grupos se recoge en la tabla I. No se encontraron diferencias significativas en la evolución de la media del volumen de dieta consumido por cada grupo.

En la figura 1 se muestran los valores de MDA en la retina. En el grupo etanol se encontraron valores de MDA mayores que en el grupo control (etanol $1,42 \mathrm{DE} 0,17$, control $0,70 \mathrm{DE} 0,04 \mathrm{nmol} / \mathrm{mg}$ prote-

Tabla I. El volumen medio de dieta ingerida de los distintos grupos

\begin{tabular}{lc}
\hline & Volumen dieta ml/día (media $\pm \mathrm{DE})$ \\
\hline Control & $71,78 \mathrm{DE} 12,6$ \\
Control + Ebselen & $68,84 \mathrm{DE} 11,8$ \\
Etanol & $69,52 \mathrm{DE} 9,8$ \\
Etanol + Ebselen & $70,23 \mathrm{DE} 8,7$ \\
\hline \hline
\end{tabular}

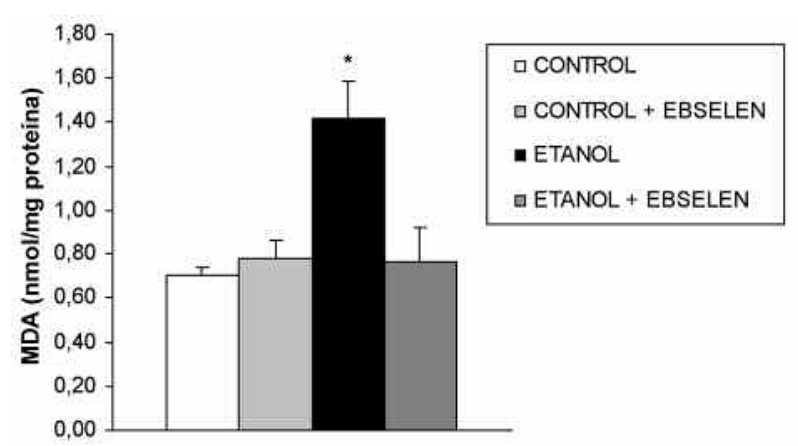

Fig. 1: Estudio de las concentraciones de MDA (nmol/mg proteína) en retina de los distintos grupos tras 6 semanas de experimento. $* p<0,05$ frente a todos los demás grupos. 
ína), mientras que la administración de ebselen disminuyó estos valores, igualándolos a los valores control (etanol+ebselen 0,76 DE 0,16, control+ebselen $0,78 \mathrm{DE} 0,08 \mathrm{nmol} / \mathrm{mg}$ proteína).

Los valores de GSH en las muestras obtenidas vienen expresados en la figura 2. La concentración de GSH disminuyó en la retina de animales alcohólicos (etanol 12,18 DE 2,37, control 23,38 DE 1,69 $\mathrm{nmol} / \mathrm{mg}$ proteína). El tratamiento de los animales alcohólicos con ebselen aumentó los valores de GSH hasta igualarlos a los valores control (etanol+ebselen 19,26 DE 2,88, control+ebselen 21,06 DE 3,45 nmol/mg proteína).

En la figura 3 se observan los valores de la amplitud de la onda b del ERG, que se encuentra significativamente disminuida en los animales alcohólicos tras 6 semanas de dieta alcohólica (etanol 126,0 DE 8,9, control 163,7 DE 12,7 $\mu \mathrm{V}$ ). El tratamiento con ebselen consigue restaurar esta reducción en la amplitud de la onda b (etanol+ebselen 150,0 DE 9,0 , control+ebselen 191,7 DE 12,8 $\mu \mathrm{V}$ ). No se

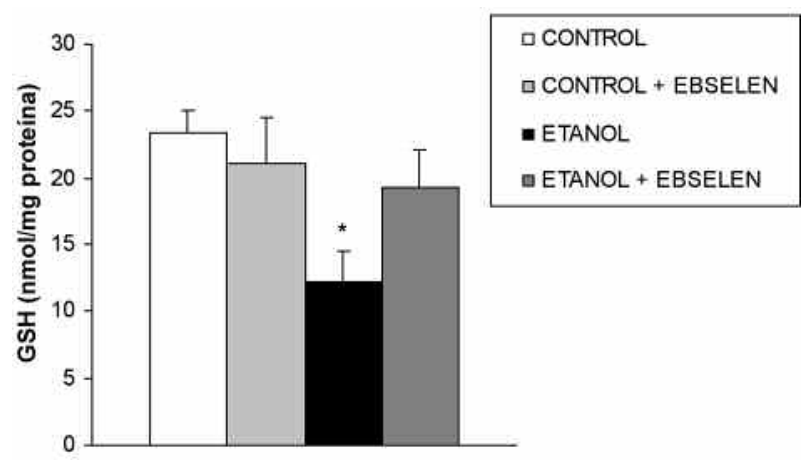

Fig. 2: Estudio del contenido de GSH (nmoles/mg proteína) en retina tras 6 semanas de experimento. $* p<$ 0,05 frente a todos los grupos.

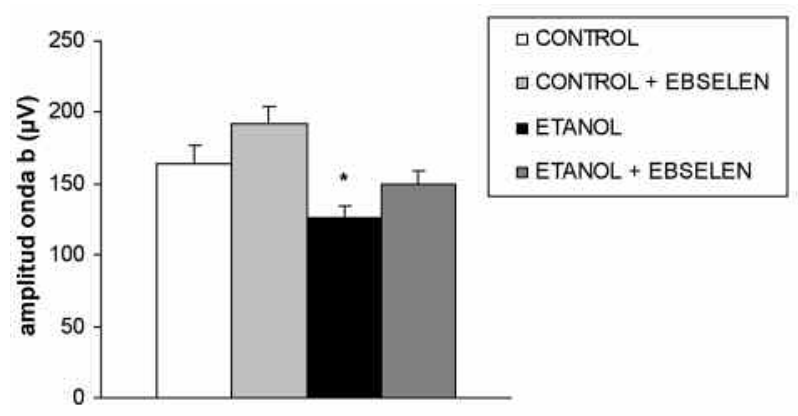

Fig. 3: Amplitud de la onda b del electrorretinograma de los distintos grupos. $* p<0,05$ frente a todos los otros grupos. encontraron diferencias significativas en la amplitud de la onda a en ninguno de los grupos estudiados (datos no mostrados).

\section{DISCUSIÓN}

Durante años no se ha aceptado la existencia de una verdadera neuropatía óptica alcohólica, negándose un efecto tóxico intrínseco del etanol. La pérdida visual que se produce en muchos alcohólicos se ha englobado generalmente en un cuadro conocido como ambliopía tabaco-alcohólica, atribuyéndose un efecto sinérgico del consumo de alcohol con el tabaco, con carencias vitamínicas secundarias a una nutrición deficiente, que conduce a una pérdida en la visión central (19). Además, se ha descrito recientemente que la ambliopía tabaco-alcohólica causa daño a la propia retina y no solamente al nervio óptico (20).

Se ha demostrado recientemente en modelos experimentales de administración crónica de etanol, un aumento de la concentración de productos de la peroxidación de lípidos en el nervio óptico (9). El hecho de que las membranas de los segmentos externos de los bastones contengan una concentración muy alta de ácidos grasos poliinsaturados de cadena larga (21), las hace particularmente susceptibles al daño oxidativo.

Los resultados obtenidos en el presente trabajo en homogenado de ojo sin cristalino de ratas alcohólicas crónicas, donde la única diferencia con el grupo control es el alcohol administrado en la dieta, muestran un incremento de los productos derivados de la peroxidacion lipídica (MDA), así como el descenso de los niveles de antioxidantes endógenos (GSH). Estas son evidencias que apoyan el papel del estrés oxidativo en patologías oculares asociadas al alcohol. Es un hecho bien documentado en la bibliografía que el $97 \%$ de los metabolitos del homogenado de ojo sin cristalino corresponden a la retina (14). Las elevadas concentraciones de MDA en los ojos de las ratas alcohólicas confirman la importancia de la peroxidación lipídica en el daño causado por la ingesta crónica del etanol. Hay un consenso general acerca de que las determinaciones de MDA por HPLC son marcadores efectivos de la implicación del estrés oxidativo en una condición patológica y son útiles para evaluar el efecto de tratamientos con antioxidantes (22). Los datos presentados en este trabajo demuestran una normalización 
de los niveles de MDA y GSH en ratas alcohólicas cuando se administra ebselen junto con la dieta, sugiriendo una posible vía de tratamiento para evitar daños causados por el estrés oxidativo como consecuencia de la ingesta crónica del etanol.

Electrorretinográficamente, en el grupo etanol se obtiene una amplitud media de la onda b de 120,0 DE $8,9 \mu \mathrm{V}$, prácticamente una reducción del $25 \%$ con respecto al valor control. Esta importante disminución en la amplitud de la onda b concuerda con otro estudio que ha demostrado que los cambios electrorretinográficos en pacientes con ambliopía tabaco-alcohólica se originan generalmente en las capas de células ganglionares y nuclear interna de la retina (20). Nuestros datos sugieren que además del nervio óptico, la propia retina esta afectada por el consumo crónico de etanol. La realización del ERG permite relacionar los parámetros de estrés oxidativo con la integridad funcional de la retina. Los resultados presentados apuntan a que existe una patología ocular, concretamente en la retina, que es atribuible a la ingesta crónica del etanol como único factor etiológico.

La administración de ebselen a las ratas alcohólicas produjo una recuperación hasta valores control de las amplitudes de la onda b en el ERG, además de una normalización de los parámetros del estrés oxidativo. El uso de ebselen, un agente con actividad similar a la glutation peroxidasa y secuestrante de peroxinitritos (3), podría considerarse como un método adecuado para el tratamiento de las alteraciones causadas por el etanol observadas en este trabajo. Considerando la capacidad de penetración del ebselen a través de la barrera hemato-retiniana (23), su baja toxicidad, y el hecho de que nuestro grupo ha demostrado previamente su eficacia en el tratamiento de otras patologías oculares $(9,14)$, así como para la neurotoxicidad inducida por etanol (6), los resultados presentados permiten proponer al ebselen como posible tratamiento en pacientes alcohólicos con problemas oculares. Aún así, se requieren nuevos estudios para confirmar el mecanismo de acción del ebselen en este modelo de alcoholismo crónico.

\section{BIBLIOGRAFÍA}

1. Dupont I, Bodenez P, Berthou F, Simon B, Bardou LG, Lucas D. Cytochrome P-450 2E1 activity and oxidative stress in alcoholic patients. Alcohol Alcohol 2000; 35: 98103.
2. Li SY, Gomelsky M, Duan J, Zhang Z, Gomelsky L, Zhang $X$, et al. Overexpression of aldehyde dehydrogenase-2 (ALDH2) transgene prevents acetaldehyde-induced cell injury in human umbilical vein endothelial cells: role of ERK and p38 mitogen-activated protein kinase. J Biol Chem 2004; 279: 11244-11252.

3. Sies H. Oxidative stress: oxidants and antioxidants. Exp Physiol 1997; 82: 291-295.

4. Seo JS, Yang KM, Kim JM, Min H, Kim CS, Burri BJ. Effect of chronic alcohol consumption on plasma lipid, vitamins $A$, and $E$ in Korean alcoholics. Nutr Res 2004; 24: 959-968.

5. Yang SS, Huang CC, Chen JR, Chiu CL, Shieh MJ, Lin SJ, et al. Effects of ethanol on antioxidant capacity in isolated rat hepatocytes. World J Gastroenterol 2005; 11: 7272 7276

6. Herrera DG, Yague AG, Johnsen-Soriano S, BoschMorell F, Collado-Morente L, Muriach M, et al. Selective impairment of hippocampal neurogenesis by chronic alcoholism: protective effects of an antioxidant. Proc Natl Acad Sci U S A 2003; 100: 7919-7924.

7. Cervantes-Munguía R, Espinosa-López L, Gómez-Contreras $P$, Hernández-Flores $G$, Domínguez-Rodríguez J, Bravo-Cuéllar A. Retinopatía del prematuro y estrés oxidativo. An Pediatr (Barc) 2006; 64: 126-131.

8. Miranda M, Muriach M, Johnsen S, Bosch-Morell F, Araiz J, Roma J, et al. Estrés oxidativo en un modelo de retinopatía diabética experimental: tratamiento con antioxidantes. Arch Soc Esp Oftalmol 2004; 79: 289-294.

9. Bosch-Morell F, Roma J, Marin N, Romero B, RodriguezGalietero A, Johnsen-Soriano S, et al. Role of oxygen and nitrogen species in experimental uveitis: anti-inflammatory activity of the synthetic antioxidant ebselen. Free Radic Biol Med 2002; 33: 669-675.

10. Aviñó J, Díaz-Llopis M, España E, Johnsen-Soriano S, Romero B, Marín N, et al. Estrés oxidativo en el nervio óptico de la rata inducido por la administración crónica de etanol. Arch Soc Esp Oftalmol 2002; 77: 263-268.

11. Tiegs $G$, Küsters $S$, Künstle $G$, Hentze H, Kiemer $A K$, Wendel A. Ebselen protects mice against T cell-dependent, TNF-mediated apoptotic liver injury. J Pharmacol Exp Ther 1998; 287: 1098-1104.

12. Sies H, Sharov VS, Klotz LO, Briviba K. Glutathione peroxidase protects against peroxynitrite-mediated oxidations. A new function for selenoproteins as peroxynitrite reductase. J Biol Chem 1997; 272: 27812-27817.

13. Lieber CS, DeCarli L, Rubin E. Sequential production of fatty liver, hepatitis, and cirrhosis in sub-human primates fed ethanol with adequate diets. Proc Natl Acad Sci U S A 1975; 72: 437-441.

14. Miranda M, Muriach M, Roma J, Bosch-Morell F, Genovés JM, Barcia J, et al. Estrés oxidativo en un modelo de retinopatía diabética experimental II: utilidad de agentes secuestrantes de perioxinitritos. Arch Soc Esp Oftalmol 2006; 81: 27-32.

15. Richard MJ, Guiraud P, Meo J, Favier AJ. High-performance liquid chromatographic separation of malondialdehyde-thiobarbituric acid adduct in biological materials (plasma and human cells) using a commercially available reagent. J Chromatogr 1992; 577: 9-18.

16. Romero FJ, Bosch-Morell F, Romero MJ, Jareño EJ, Romero B, Marín N, et al. Lipid peroxidation products 
and antioxidants in human disease. Environ Health Perspect 1998; 106 Suppl 5: 1229-1234.

17. Reed DJ, Babson JR, Beatty PW, Brodie AE, Ellis WW, Potter DW. High-performance liquid chromatography analysis of nanomole levels of glutathione, glutathione disulfide, and related thiols and disulfides. Anal Biochem 1980; 106: 55-62.

18. Lowry OH, Rosebrough NJ, Farr AL, Randall RJ. Protein measurement with the Folin phenol reagent. J Biol Chem 1951; 193: 265-275.

19. Carroll FD. The Etiology and Treatment of Tobacco-Alcohol amblyopia. Trans Am Ophtalmol Soc 1943; 41: $385-$ 431.

20. Behbehani R, Sergott RC, Savino PJ. Tobacco-alcohol amblyopia: a maculopathy? Br J Ophthalmol 2005; 89: 1543-1544.

21. Martin RE, Fliesler SJ, Brush RS, Richards MJ, Hopkins SA, Anderson RE. Lipid differences in rod outer segment membranes of rats with $\mathrm{P} 23 \mathrm{H}$ and S334ter opsin mutations. Mol Vis 2005; 11: 338-346.

22. Halliwell B. Oxidative stress markers in human disease: application to diabetes and to evaluation of the effects of antioxidants. In: Packer, L, Rosen P, Tritschler HJ, King GL, Azzi A. Antioxidants in Diabetes Management. New York: Marcel Dekker; 2000; 33-52.

23. Bosch-Morell F, Roma J, Puertas FJ, Marin N, Diaz-Llopis $M$, Romero FJ. Efficacy of the antioxidant ebselen in experimental uveitis. Free Radic Biol Med 1999; 27: 388-391. 